\title{
Detection of Jaagsiekte sheep retrovirus in the peripheral blood during the pre-clinical period of ovine pulmonary adenomatosis
}

\author{
Y. Liu ${ }^{1}$, Y.F. Zhang ${ }^{1}$, X.L. Sun ${ }^{1}$ and S.Y. Liu ${ }^{1,2}$ \\ ${ }^{1}$ College of Veterinary Medicine, Inner Mongolia Agricultural University, \\ Huhhot, China \\ ${ }^{2}$ Key Laboratory of Clinical Diagnosis and Treatment Technology in Animal \\ Disease, Ministry of Agriculture, Huhhot, China
}

Corresponding author: S.Y. Liu

E-mail: liushuying_imau@126.com

Genet. Mol. Res. 15 (3): gmr.15038521

Received February 1, 2016

Accepted April 8, 2016

Published August 29, 2016

DOI http://dx.doi.org/10.4238/gmr.15038521

Copyright $(2016$ The Authors. This is an open-access article distributed under the terms of the Creative Commons Attribution ShareAlike (CC BY-SA) 4.0 License.

\begin{abstract}
The envelope protein (Env) of the Jaagsiekte sheep retrovirus (JSRV) is known to be a unique oncoprotein responsible for inducing ovine pulmonary adenocarcinoma (OPA). The objective of this study was to prepare a specific monoclonal antibody $(\mathrm{mAb})$ against the JSRV Env protein using bioinformatic analysis. According to the structure and epitope prediction results of JSRV Env, the JSRV-Env ${ }_{572-615}$ antigen was prepared via peptide synthesis (amino acid sequence 572615 , denoted as JSRV-Env ${ }_{572-615}$ ). BALB/c mice were immunized to prepare the anti-JSRV-Env ${ }_{572-615} \mathrm{mAb}$. Spleen cells were fused with SP2/0 myeloma cells after being screened by indirect ELISA and cloned by limiting dilution. The specificity of $\mathrm{mAb}$ was evaluated by western blot analysis and immunohistochemistry assays. Western blot results showed that the JSRV Env protein was able to bind to mAb with high
\end{abstract}


specificity. Immunohistochemistry assays demonstrated that the mAb was able to recognize JSRV Env in adenomatous hyperplasia of the lung. Furthermore, JSRV was detected in peripheral blood leukocytes during the pre-clinical period of OPA in 2 of the 25 sheep using this newly synthesized $\mathrm{mAb}$. Therefore, this $\mathrm{mAb}$ may be a useful tool for the detection of JSRV in sheep.

Key words: Epitope prediction; Jaagsiekte sheep retrovirus; Monoclonal antibody; Pre-clinical period detection

\section{INTRODUCTION}

Jaagsiekte sheep retrovirus (JSRV) is the etiologic agent of ovine pulmonary adenocarcinoma (OPA) (Palmarini and Fan, 2001, 2003; Leroux et al., 2007). OPA is a significant disease in many sheep-rearing countries in Europe, Africa, Asia, and the Americas (Sharp and DeMartini, 2003). Its latent period lasts from several months to years (Griffiths et al., 2010). Production of copious amounts of fluid from the lung is a symptom of OPA. The fluid has a frothy, clear, milky, or at times pinkish appearance, and drains from the sheep's nostrils when the sheep lowers its head and lifts its rear end (Hofacre and Fan, 2010). However, this lung fluid is not easily observed in many cases, which makes it difficult to carry out preventive measures and control virus transmission in a timely manner. Currently, postmortem examinations and histopathological studies are considered the most reliable ways to diagnose OPA (Azizi et al., 2014). However, these methods are limited in detecting OPA in live animals. PCR of blood samples can be employed to detect JSRV-infected cells during the pre-clinical period (González et al., 2001; Salvatori et al., 2004; Caporale et al., 2005; De Las Heras et al., 2005). However, this method is limited in its ability to identify only a few infected animals (Lewis et al., 2011).

Sheep are immune-tolerant of JSRV, as the structure of JSRV resembles endogenous retroviruses (Summers et al., 2002; Spencer et al., 2003). The sheep genome contains approximately 30 copies of the endogenous retrovirus enJSRV, which is highly related to the exogenous betaretrovirus exJSRV. The amino acid and nucleotide sequence of enJSRV demonstrate $90-98$ and $85-89 \%$ homology to that of exJSRV, respectively (Palmarini et al., 2000; Carlson et al., 2003; Arnaud et al., 2007).

Some studies have reported that the enJSR $V$ mRNA is expressed in the immune organs of fetuses and lambs (Qi et al., 2012), which leads to the absence of JSRV-specific antibodies in infected sheep. This has restricted the development of serological diagnostic tests.

The objective of this study was to build a monoclonal antibody $(\mathrm{mAb})$ against the JSRV envelope (Env) protein, and to determine its specificity. JSRV, classified as a betaretrovirus, resembles a simple retrovirus. Its genome contains essential genes of retroviruses such as gag, pro, pol, and env. The env gene encodes the surface and transmembrane domains of the Env protein, which is located at the outermost layer of the virus (Griffiths et al., 2010; Hofacre and Fan, 2010). This domain has been shown to be the dominant oncoprotein for inducing cell transformation both in vitro (Cousens et al., 2007; Liu and Miller, 2007) and in vivo (Wootton et al., 2005; Caporale et al., 2006). Even though exJSRV and enJSRV demonstrate $89 \%$ homology in nucleotide sequence, there are distinct differences at the 3 '-end of the env gene between exJSRV and enJSRV (Bai et al., 1999; Cousens et al., 1999; Palmarini et al.,

Genetics and Molecular Research 15 (3): gmr.15038521 
2000). Therefore, the cytoplasmic tail of the transmembrane portion of JSRV Env (amino acid sequence 572-615, denoted as JSRV-Env $572-615$ ) encoded by the sequence at the 3'-end of the env gene was used in this study for $\mathrm{mAb}$ synthesis. We hypothesized that the $\mathrm{mAb}$ designed with JSRV-Env ${ }_{572-615}$ could be an effective method for detecting JSRV in OPA-affected animals.

\section{MATERIAL AND METHODS}

\section{Materials}

Tumoral lung tissues infected with JSRV were collected from a sheep-breeding farm in Siziwangqi of Hohhot, Inner Mongolia Province, China. Tumoral neoplasms were confirmed by autopsy, histopathological examinations, and hemi-nested PCR (González et al., 2001). Tissue samples were placed into individual vials, immediately frozen in liquid nitrogen, and stored at $-80^{\circ} \mathrm{C}$ for protein and DNA extraction. The adjacent areas on the same tissues were stored in $10 \%$ buffered formalin for subsequent hematoxylin-eosin (H\&E) staining and immunohistochemistry. Peripheral blood leukocytes (PBLs) were collected from non-diseased sheep in OPA-affected flocks. The PBLs were isolated by centrifugation following lysis of erythrocytes. The resulting cell pellets were stored at $-80^{\circ} \mathrm{C}$. Negative control samples of PBLs were obtained from healthy sheep imported from Australia. These PBLs were identified with hemi-nested PCR (González et al., 2001). Negative control samples of lung tissues were identified via histopathological examination and hemi-nested PCR (González et al., 2001).

\section{Ethics statement}

All animal procedures were approved by the Inner Mongolia Agriculture University Animal Care and Use Committee in accordance with the National Animal Care Standard (GB 14925-2001). All efforts were made to minimize animal suffering.

\section{Analysis of the JSRV Env protein}

The amino acid sequence of JSRV Env (No. JQ837489) was obtained from the GenBank (http://www.ncbi.nih.gov/genbank/). The tertiary structure of JSRV Env was predicted by the iterative-TASSER (I-TASSER) approach (Yang et al., 2015). In I-TASSER, threading and homology modeling methods were used to predict the tertiary structure. Subsequently, adjustments and compilations were conducted using the RasMol version 2.7.0.1 software (http://www.openrasmol.org/software/rasmol/README.html). The C-score was a confidence score for estimating the quality of the predicted models. The higher the C-score used in a model, the higher the confidence, and vice versa. The B-cell epitopes of the envelope protein were predicted using Bcepred and ABCpred softwares (Sollner et al., 2008). Based on the features of the software, the thresholds for hydrophilicity, polarity, flexibility, exposed surface, accessibility, and antigenic propensity area were set at 2.0, 2.3, 1.9, 2.4, 2.0, and 1.8, respectively.

\section{Preparation of monoclonal antibodies against JSRV-Env ${ }_{572-615}$}

According to the structure and epitope prediction results of JSRV Env, the JSRVEnv $_{572-615}$ antigen was prepared by peptide synthesis, and connected with the keyhole limpet

Genetics and Molecular Research 15 (3): gmr.15038521 
hemocyanin (KLH) and bovine serum albumin (BSA) to improve antigenicity (Chinese Peptide Company, China). BALB/c mice (5 weeks of age) were injected subcutaneously with $100 \mu \mathrm{g}$ KLH-JSRV-Env ${ }_{572-615}$ peptide emulsified with isopycnic complete Freund's adjuvant in the first immunization. The incomplete Freund's adjuvant was used in the two subsequent booster shots at 2-week intervals. A final tail intravenous immunization with the KLHJSRV-Env $_{572-615}$ peptide alone was administered 3 days before cell fusion. Splenocytes from $\mathrm{BALB} / \mathrm{c}$ mice immunized with KLH-JSRV-Env ${ }_{572-615}$ were harvested and subsequently fused with SP2/0 myeloma cells (10:1 ratio) using 50\% PEG1450. The cells were cultured in DMEM containing $10 \%$ fetal calf serum and $10 \%$ HAT for 14 days. Half of this medium was replaced with fresh medium containing 10\% HAT/HT at days 3, 6 and 9. Hybridoma supernatants were screened for reactivity with enzyme-linked immunosorbent assay (ELISA), and positive clones were sub-cloned three times by the limiting dilution method. The titer of the antibody was verified by ELISA. Briefly, BSA-JSRV-Env ${ }_{572-615}$ was diluted (concentration of $0.02 \mathrm{mg} / \mathrm{mL}$ ), coated on 96 -well plates $\left(100 \mu \mathrm{L} /\right.$ well), and stored at $4{ }^{\circ} \mathrm{C}$ overnight. It was then washed four times with PBST before $200 \mu \mathrm{L} 5 \%$ skim milk was added. The plate was incubated at $37^{\circ} \mathrm{C}$ for $1 \mathrm{~h}$. After washing four times with PBST, it was kept at $37^{\circ} \mathrm{C}$ for another hour with $100 \mu \mathrm{L}$ culture supernatants and ascites at different concentrations (from 1:800 to $1: 12,800$ and from 1:100 to 1:1,000,000, respectively). The culture supernatants and ascites of SP2/0 myeloma cells were used as negative controls. Next, $100 \mu$ L HRP-conjugated goat anti-mouse IgG (EarthOx, USA) at 1:10,000 dilution was added to the wells, and the plate was incubated at $37^{\circ} \mathrm{C}$ for $1 \mathrm{~h}$. Freshly prepared 3, 3', 5, 5'-tetramethylbenzidine (TMB, TIANGEN, Beijin, China) solution was used as the substrate to detect peroxidase activity. Color development was terminated after 15 min using isopycnic $2 \mathrm{mM} \mathrm{H}_{2} \mathrm{SO}_{4}$. Light absorbance was measured at $450 \mathrm{~nm}$ using a microplate reader (Bio-Rad, Hercules, CA, USA). The absorbance values at $450 \mathrm{~nm}$ from serum samples of 25 healthy mice were measured to calculate the cut-off value of ELISA.

\section{Purification and subclass identification of monoclonal antibodies against JSRV- Env $_{\text {572-615 }}$}

The crude ascitic fluid was collected from the peritoneal cavities of atolin primed BALB/c mice via centrifugation (4200 $g$ for $5 \mathrm{~min}$ ). The globulin fraction was isolated via two rounds of selective precipitation with octanoic acid (2.5\% saturation) and ammonium sulfate (45\% saturation). Following the final precipitation, the proteins were dissolved in $\mathrm{pH}$ 7.4 PBS solution. The mAbs were desalted by dialysis, and were further purified using the Protein G Spin Purification Kit (PIERCE, USA). The purified mAbs were analyzed by SDSPAGE and stored at $-80^{\circ} \mathrm{C}$ for further use. The subclass of $\mathrm{mAb}$ was determined by the Mouse Monoclonal Antibody Isotype Assay Kit (Sigma, St. Louis, MO, USA).

\section{Western blot analysis and immunohistochemistry assay}

The specificity of $\mathrm{mAb}$ was evaluated by western blot analysis and immunohistochemistry assay. The total protein from lung tissue of OPA-affected sheep was extracted using the total protein extraction kit (Shanghai, China). Samples were loaded on a $10 \%$ SDS-PAGE, and were transferred to a nitrocellulose membrane. For western blot analysis, membranes were successively blocked with $5 \%$ BSA at $4^{\circ} \mathrm{C}$ overnight. Membranes

Genetics and Molecular Research 15 (3): gmr.15038521 
were incubated with $\mathrm{mAb}$ (1:600 dilution) for $1 \mathrm{~h}$, washed five times with TBST, and treated

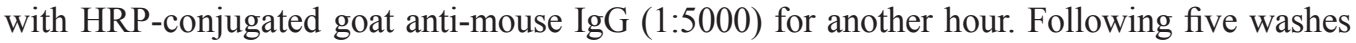
with TBST, protein visualization was carried out with 3,3'-diaminobenzidine-4HCl (DAB, Max Vision $^{\mathrm{TM}}$, Fujian, China).

Immunohistochemistry staining was also applied to assess the specificity of the mAb. Thick paraffin sections $(4 \mu \mathrm{m})$ were freshly prepared with lung tissues from the JSRV-infected sheep, and were incubated in 3\% hydrogen peroxide at room temperature for 20 min to block endogenous peroxidase activity. The sections were boiled in $10 \mathrm{mM}$ citrate buffer, $\mathrm{pH} 6.0$, for $30 \mathrm{~min}$, and were blocked for $30 \mathrm{~min}$ with $10 \%$ bovine serum. Samples were incubated with $\mathrm{mAb}\left(1: 600\right.$ dilution) at $4^{\circ} \mathrm{C}$ overnight. After five PBS washes, HRP-labeled goat anti-mouse IgG (Max Vision ${ }^{\mathrm{TM}}$ ) was used to detect immunological reactions. Normal lung tissues were used as negative control.

\section{Detection of JSRV in non-diseased sheep from OPA-affected flocks}

The PBLs of the 25 non-diseased sheep from OPA-affected flocks were isolated and stored at $-80^{\circ} \mathrm{C}$. For western blotting, PBLs were suspended in $2.5 \mathrm{~mL}$ blood with $40 \mu \mathrm{L}$ PBS. The supernatant was loaded on $10 \%$ polyacrylamide gels, and were subsequently transferred to nitrocellulose membranes for western blotting. JSRV-positive sheep were further verified with immunohistochemistry and hemi-nested PCR. However, due to the lack of experimental appropriations, JSRV-negative sheep were only verified by hemi-nested PCR but not postmortem examination. Genomic DNA of lung tissues was extracted with the genomic DNA extraction kit according to manufacturer instructions (TIANGEN).

\section{RESULTS}

\section{Analysis results of the JSRV Env protein}

According to the information from GenBank (No. JQ837489), JSRV Env is composed of 615-amino acid residues. The tertiary structure of JSRV Env was predicted by I-TASSER. These tertiary structure results are displayed in the space-filling model illustrated in Figure 1. The server recommended five tertiary structure models, which are all presented as space-filling models (Figure 1A-E). The C-score is a confidence score for estimating the quality of the predicted models, and usually ranges from -5 to 2 . The $\mathrm{C}$-scores of the five models were $-0.45,-1.45,-3.63,-4.30$, and -2.75 . The higher the $\mathrm{C}$-score is for a model, the higher the confidence for that specific structure. Different colors represent different secondary structures: pink, $\alpha$ helix; blue, $\beta$ turn; yellow, $\beta$-fold, and gray, random coil. The positions 572-615 were displayed in the space-filling model (Figure 1F-J). Although there are differences in the five tertiary structure models, they all have open architectures based on random coils and $\beta$ turns, and show good spatial flexibility, scalability, as well as accessibility.

The B-cell epitopes of the envelope protein were predicted using both the Bcepred and $\mathrm{ABCpred}$ programs. The prediction results of $\mathrm{B}$-cell epitopes and the cytoplasmic tail are listed in Tables 1 and 2, respectively. These predictions suggest that the cytoplasmic tail of JSRV envelope protein may be the dominant antigen-binding region.

Genetics and Molecular Research 15 (3): gmr.15038521 


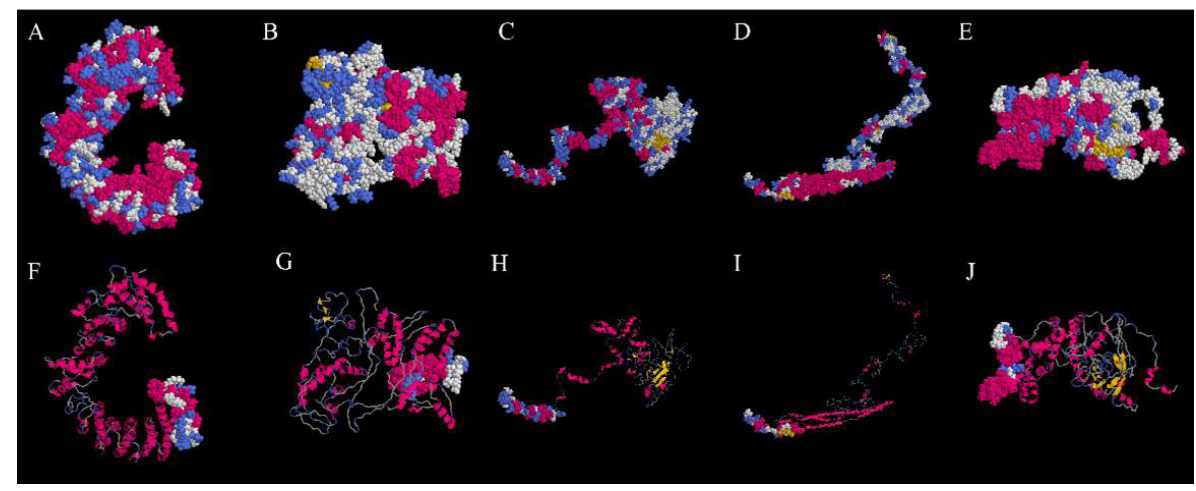

Figure 1. Tertiary structure prediction for the JSRV Env and JSRV-Env ${ }_{572-615}$ protein. Tertiary structure of JSRV Env was predicted by the I-TASSER online server. The server recommended five tertiary structure models, which are presented here as space-filling models (A-E). Different colors represent different secondary structures: pink, $\alpha$ helix; blue, $\beta$ turn; yellow, $\beta$-fold; gray, random coil. These models depict the area located at positions of 572-615-amino acid residues of JSRV Env (F-J).

Table 1. Results of the analysis of B-cell epitopes on the cytoplasmic tail using the Bcepred software.

\begin{tabular}{l|c|l|l}
\hline No. & Start-end position & Amino acid sequence & Physical and chemical properties \\
\hline 1 & $604-615$ & KNKERGDAGDDP & Hydrophilic \\
\hline 2 & $576-582$ & RDFLKMR & Surface accessibility \\
\hline 3 & $568-597$ & LHMKYRNMLQHQ & Surface accessibility \\
\hline 4 & $600-613$ & MELLKNKERGDAGD & Surface accessibility \\
\hline 5 & $600-609$ & MELLKNKERG & Flexibility \\
\hline 6 & $604-607$ & KNKE & Polarity \\
\hline 7 & $576-593$ & RDFLKMRVEMLHMKYRNM & Exposed surface \\
\hline 8 & $595-614$ & QHQHLMELLKNKERGDAGDD & Exposed surface \\
\hline 9 & $550-575$ & LLGLGILVFIIIVVILIFPCLVRGMV & Antigen propensity \\
\hline
\end{tabular}

Table 2. Results of the analysis of B-cell epitopes on the cytoplasmic tail using the ABCpred software.

\begin{tabular}{l|c|l|c}
\hline No. & Start-end position & Amino acid sequence & Score \\
\hline 1 & $600-615$ & MELLKNKERGDAGDDP & 0.95 \\
\hline 2 & $572-587$ & RGMVRDFLKMRVEMLH & 0.83 \\
\hline 3 & $581-596$ & MRVEMLHMKYRNMLQH & 0.74 \\
\hline 4 & $562-577$ & VVILIFPCLVRGMVRD & 0.62 \\
\hline
\end{tabular}

\section{Purification and subclass identification of the JSRV Env-specific mAb}

The purification results showed that the molecular weight of the light and heavy chains were approximately 25 and $55 \mathrm{kDa}$ (Figure 2A), respectively. Purified mAbs were successfully obtained, which could meet the requirements of the follow-up experiments.

After three rounds of sub-cloning, hybridoma supernatants, which reacted with BSAJSRV-Env $_{572-615}$, were used to screen antibody-secreting hybridoma cells by indirect ELISA. One clone showed strong and specific reactivity to the JSRV-Env ${ }_{572-615}$ protein, and was selected and designated as $8 \mathrm{E} 5$. Isotype results showed that the $\mathrm{mAb}$ produced by this clone (denoted as $\mathrm{mAb} 8 \mathrm{E} 5$ ) belonged to the IgG2a subtype, and the light chains were type $\kappa$ (Figure 3A). In this study, the anti-JSRV-Env ${ }_{572-615}$ antibodies secreted by other hybridoma cells were also examined, but they all were classified as $\operatorname{IgM}$ (data not shown).

Genetics and Molecular Research 15 (3): gmr.15038521 

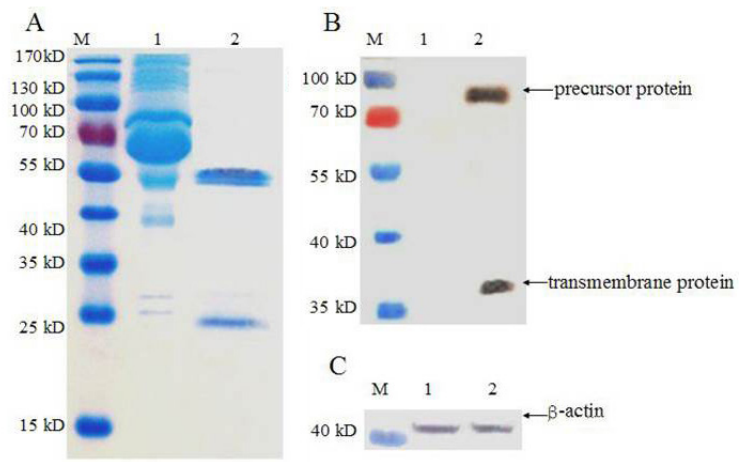

Figure 2. Purification of ascites and western blot analysis of mAb 8E5. A. mAbs from ascites purified and analyzed by SDS-PAGE. Lanes: $M$, protein molecular weight markers; 1 , unpurified ascites; 2, purified ascites. B. Western blots showing that $\mathrm{mAb} 8 \mathrm{E} 5$ specifically recognized the natural precursor protein of JSRV Env and the transmembrane protein. Lanes: $M$, protein molecular weight markers; 1 , total protein from lung tissue of OPA-non-affected sheep (negative control); 2 , total protein from lung tissue of OPA-affected sheep. C. $\beta$-actin was used as the internal control.

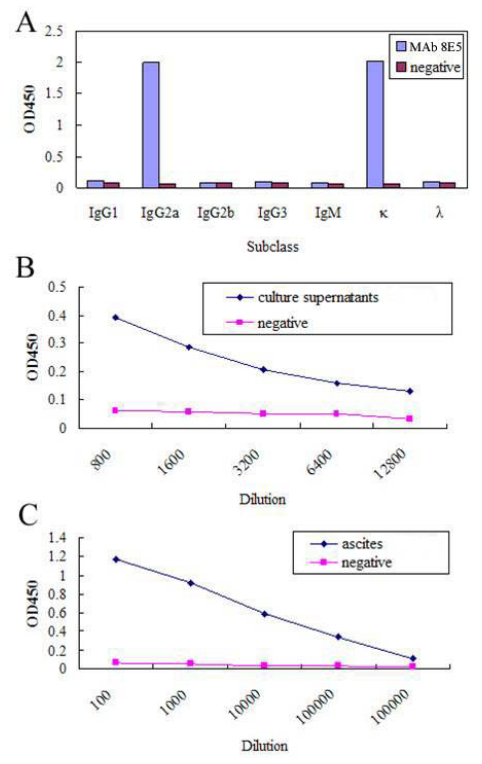

Figure 3. Subclass and titer analysis of mAb 8E5. Isotyping of mAb 8E5 (A). Titration of mAb 8E5 by ELISA. Hybridoma supernatants and ascites of mAb 8E5 were titered by ELISA. Supernatant from SP2/0 cells were used as negative control. Dilution titers of mAb 8E5 in culture supernatants (B) and ascites (C) were 6400 and 100,000, respectively.

\section{Specificity determination of mAb 8E5}

The titer of the mAb 8E5 was checked by indirect ELISA analysis. According to the criterion of ELISA (cut-off value $=0.128-0.143$ ), the titer of culture supernatants and ascites were 1:6400 and 1:100,000, respectively (Figure 3B and C). Western blot results showed that $\mathrm{mAb} 8 \mathrm{E} 5$ could specifically recognize the natural precursor protein of the JSRV Env and transmembrane protein (Figure 2B). Immunohistochemistry was performed to assess the 
specificity of the mAb $8 \mathrm{E} 5$. The results showed that positive signals were mainly present in adenomatous hyperplasia focal of the lung tissue (Figure 4A and B); this was consistent with our western blot results. Normal lung tissues (Figure 4C) and HBsAg mAb targeted against OPA-affected lung tissue (Figure 4D) were used as negative controls.

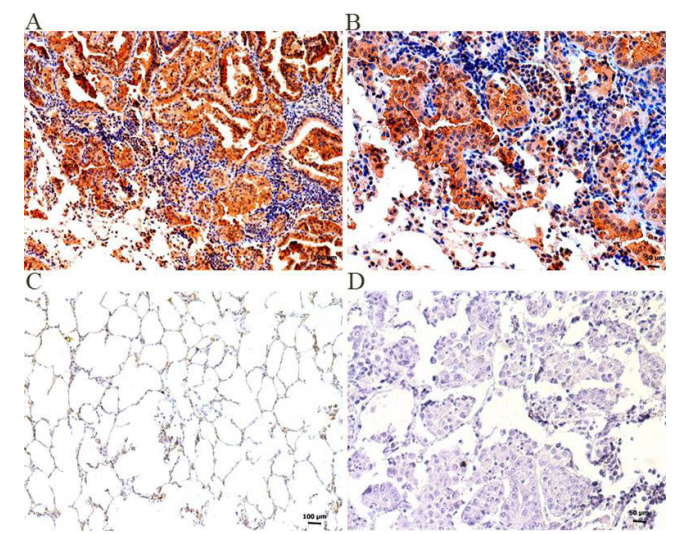

Figure 4. Specificity identification of $m A b 8 E 5$ by immunohistochemistry. Lung tumors of sheep infected with JSRV were stained with mAb 8E5 (A, B). Normal lung tissues (C) and HBsAg mAb against OPA-affected lung tissue (D) stained with $\mathrm{mAb} 8 \mathrm{E} 5$ were used as negative control. [A $(\mathrm{bar}=100 \mu \mathrm{m}) ; \mathrm{B}(\mathrm{bar}=50 \mu \mathrm{m}) ; \mathrm{C}(\mathrm{bar}=100 \mu \mathrm{m}) ; \mathrm{D}(\mathrm{bar}=50 \mu \mathrm{m})$ ].

\section{Detection of non-diseased sheep from OPA-affected flocks with mAb 8E5}

The PBLs of 25 non-diseased sheep from OPA-affected flocks were detected with mAb $8 \mathrm{E} 5$ by western blot. Two of the 25 sheep were positive for JSRV (Figure 5). These two sheep (Animal ID SZWQ1101 and SZWQ1362, respectively) were euthanized, and clinical autopsy was carried out. Lesions were found in the lungs in both JSRV-positive sheep. Lung tissue sections were probed with mAb 8E5. Tumor-like excrescences were discovered in the lung tissue where immunostaining results also indicated positive signals (Figure 6). The heminested PCR products (133 bp) of lung tissues were visualized by electrophoresis. Interestingly, the hemi-nested PCR of PBLs from both sheep showed negative results (Figure 7).

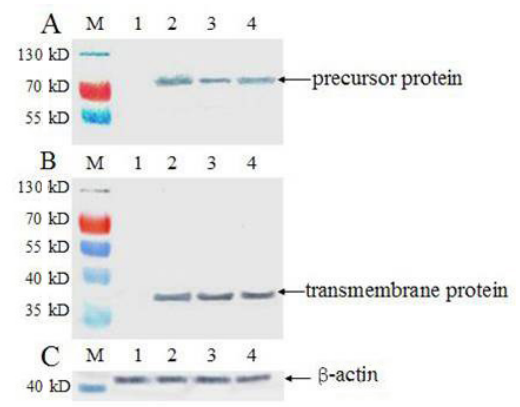

Figure 5. Detection of the JSRV Env protein in PBLs of the non-diseased sheep from OPA-affected flocks with $\mathrm{mAb}$ 8E5. The precursor protein of JSRV Env (A) and the transmembrane protein (B) in PBLs were both probed with mAb 8E5. Lanes: $M$, protein molecular weight markers; 1, PBLs from normal sheep (negative control); 2, PBLs from OPA-affected sheep (positive control); 3, PBLs from sheep SZWQ1101; 4, PBLs from sheep SZWQ1362. $\beta$-actin was used as the internal control (C).

Genetics and Molecular Research 15 (3): gmr.15038521 


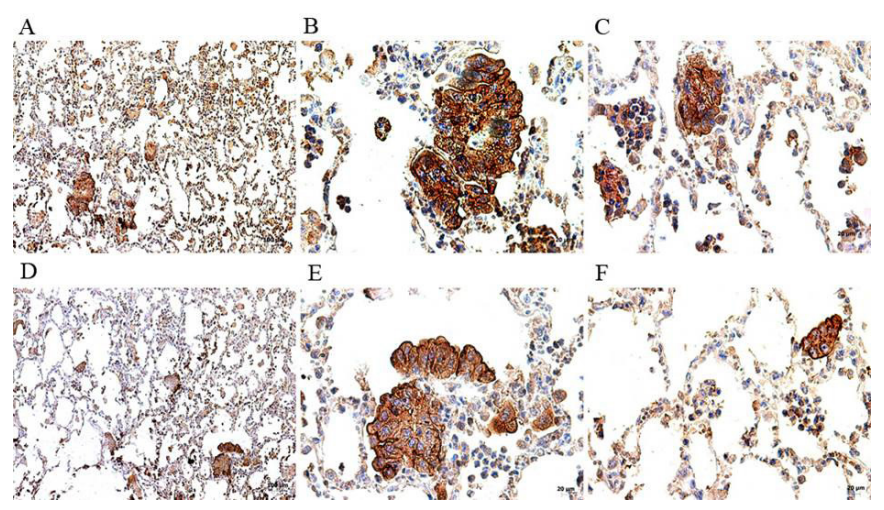

Figure 6. Detection of the JSRV Env protein in lung tissue of JSRV-positive sheep with mAb $8 \mathrm{E} 5$ by immunohistochemistry. (A-C) represent lung tissue of sheep SZWQ1101 [A (bar $=100 \mu \mathrm{m}), \mathrm{B}(\mathrm{bar}=50 \mu \mathrm{m}), \mathrm{C}(\mathrm{bar}$ $=50 \mu \mathrm{m})] ;(\mathbf{D}-\mathbf{F})$ represent lung tissue of sheep SZWQ1362 [D $($ bar $=100 \mu \mathrm{m}), \mathrm{E}(\mathrm{bar}=50 \mu \mathrm{m}), \mathrm{F}(\mathrm{bar}=50 \mu \mathrm{m})]$.

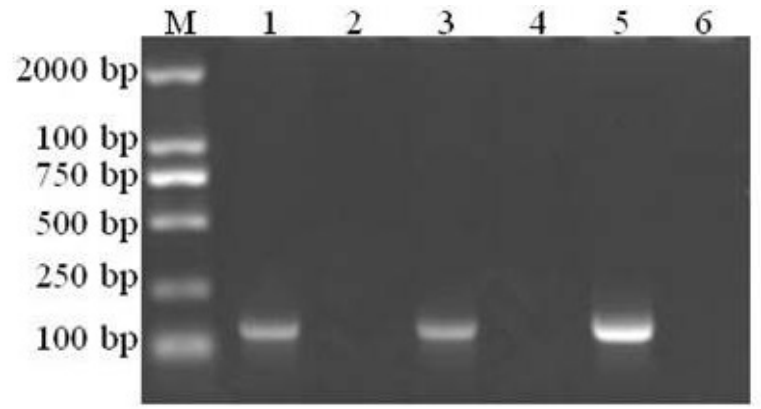

Figure 7. Identification of JSRV-positive sheep by hemi-nested PCR. Lanes: M, DL2000 DNA Marker; 1 and 2, lung issue and PBLs from sheep SZWQ1101, respectively; 3 and 4, lung issue and PBLs from sheep SZWQ1362, respectively; 5 , positive control; 6 , negative control.

\section{DISCUSSION}

The five predicted tertiary structure models all have open architectures based on random coils and $\beta$ turns. These structures showed that the JSRV-ENV ${ }_{572-615}$ protein has good spatial flexibility, scalability, and accessibility. These characteristics were conducive to antigen-antibody binding (Sollner et al., 2008). The bioinformatic analysis of JSRV Env provided the necessary experimental data for the identification and screening of epitopes, and could enhance the accuracy and efficacy of antibody synthesis. Based on the results of our bioinformatic analysis, the 572-615-amino acid region was selected as the candidate antigen for synthesis of the JSRV Env mAb. Results from the epitope analysis were also in agreement with the bioinformatic analysis.

A few studies have previously detected JSRV capsid protein as well as orf-x, gag and endogenous JSRV sequences in human tissues (Rocca et al., 2008). However, there is no clear consensus on the correlation between JSRV and human lung cancer (Yousem et al., 2001; De Las Heras et al., 2007; Hopwood et al., 2010). Interestingly, one study has reported that a subset of human lung cancers expressed an antigen that could react with a JSRV Envspecific monoclonal antibody. Furthermore, they reported that exogenous JSRV-like env and 
gag sequences from tumor samples can be amplified (Linnerth-Petrik et al., 2014). In addition, a number of epidemiological studies indicated that workers in abattoirs and meat processing plants show increased risk of developing lung cancer, which may be due to exposure to oncogenic viruses such as JSRV and bovine papilloma virus in these animals (McLean and Pearce, 2004; Johnson, 1994, 2011). This has led to the speculation that JSRV could be linked to a subset of human lung cancers (Sun et al., 2007).

In this study, a JSRV Env-specific mAb (8E5) was generated using hybridoma technology. Exogenous virus-specific hemi-nested PCR in the U3 region of JSRV LTR was used to assess the lung tissue and PBLs of two possible OPA-affected sheep. Although PBL samples from these two sheep were positive for JSRV when analyzed by western blot, hemi-nested PCR results of PBLs were negative. This could be explained by the low proportion of infected cells in blood, resulting in low amount of JSRV DNA and increased DNA degradation following extraction. We did not find copious amounts of frothy liquid in the trachea, but a tiny lesion was observed on the surface of the lung. These clinical symptoms are known to be a sign of pre-clinical period of OPA. We confirmed that these two sheep have been affected by OPA, as tumor-like excrescences were observed during histopathological examination by IHC, which is the most reliable method for OPA diagnosis (Azizi et al., 2014). The results reported here indicate that mAb could be used for detection of JSRV in PBLs of sheep during the pre-clinical period of OPA. However, it must be stated that a negative scan cannot provide a guarantee that the animal is free of JSRV infection or early OPA.

We have shown that mAb synthesized based on the anti-cytoplasmic tail (CT) of JSRV Env was specific to JSRV. This is due to the fact that the CT region is distinct different between exogenous JSRV and endogenous JSRV (Bai et al., 1999; Cousens et al., 1999; Palmarini et al., 2000). Using anti-CT mAb as the detection antibody is important for further exploration of early differential diagnostic methods for OPA. Hence, high-throughput and next-generation etiologic detection approaches at the protein level might be helpful for investigation of OPA and JSRV-like viruses, and have profound implications for the prevention, diagnosis and therapy of such diseases.

\section{Conflicts of interest}

The authors declare no conflict of interest.

\section{ACKNOWLEDGMENTS}

Research supported by the Project of the National Natural Science Foundation of China (\#31160493, \#31360597), the Inner Mongolia Science and Technology Innovation Fund Project guide (\#20130224), and the Doctoral Fund of Ministry of Education doctoral projects (\#20111515110008).

\section{REFERENCES}

Arnaud F, Murcia PR and Palmarini M (2007). Mechanisms of late restriction induced by an endogenous retrovirus. $J$. Virol. 81: 11441-11451.http://dx.doi.org/10.1128/JVI.01214-07

Azizi S, Tajbakhsh E and Fathi F (2014). Ovine pulmonary adenocarcinoma in slaughtered sheep: a pathological and polymerase chain reaction study. J. S. Afr. Vet. Assoc. 85: 932. http://dx.doi.org/10.4102/jsava.v85i1.932

Genetics and Molecular Research 15 (3): gmr.15038521 
Bai J, Bishop JV, Carlson JO and DeMartini JC (1999). Sequence comparison of JSRV with endogenous proviruses: envelope genotypes and a novel ORF with similarity to a G-protein-coupled receptor. Virology 258: 333-343. http:// dx.doi.org/10.1006/viro.1999.9728

Caporale M, Centorame P, Giovannini A, Sacchini F, et al. (2005). Infection of lung epithelial cells and induction of pulmonary adenocarcinoma is not the most common outcome of naturally occurring JSRV infection during the commercial lifespan of sheep. Virology 338: 144-153.http://dx.doi.org/10.1016/j.virol.2005.05.018

Caporale M, Cousens C, Centorame P, Pinoni C, et al. (2006). Expression of the jaagsiekte sheep retrovirus envelope glycoproteins is sufficient to induce lung tumors in sheep. J. Virol. 80: 8030-8037. http://dx.doi.org/10.1128/ JVI.00474-06

Carlson J, Lyon M, Bishop J, Vaiman A, et al. (2003). Chromosomal distribution of endogenous Jaagsiekte sheep retrovirus proviral sequences in the sheep genome. J. Virol. 77: 9662-9668. http://dx.doi.org/10.1128/JVI.77.17.9662$\underline{9668.2003}$

Cousens C, Minguijon E, Dalziel RG, Ortin A, et al. (1999). Complete sequence of enzootic nasal tumor virus, a retrovirus associated with transmissible intranasal tumors of sheep. J. Virol. 73: 3986-3993.

Cousens C, Maeda N, Murgia C, Dagleish MP, et al. (2007). In vivo tumorigenesis by Jaagsiekte sheep retrovirus (JSRV) requires Y590 in Env TM but not full-length orfX open reading frame. Virology 367: 413-421. http://dx.doi. org/10.1016/j.virol.2007.06.004

De Las Heras M, Ortín A, Salvatori D, Pérez de Villareal M, et al. (2005). A PCR technique for the detection of Jaagsiekte sheep retrovirus in the blood suitable for the screening of ovine pulmonary adenocarcinoma in field conditions. Res. Vet. Sci. 79: 259-264. http://dx.doi.org/10.1016/j.rvsc.2005.02.003

De Las Heras M, Murcia P, Ortín A, Azúa J, et al. (2007). Jaagsiekte sheep retrovirus is not detected in human lung adenocarcinomas expressing antigens related to the Gag polyprotein of betaretroviruses. Cancer Lett. 258: 22-30. http://dx.doi.org/10.1016/j.canlet.2007.08.006

González L, García-Goti M, Cousens C, Dewar P, et al. (2001). Jaagsiekte sheep retrovirus can be detected in the peripheral blood during the pre-clinical period of sheep pulmonary adenomatosis. J. Gen. Virol. 82: 1355-1358. http://dx.doi. org/10.1099/0022-1317-82-6-1355

Griffiths DJ, Martineau HM and Cousens C (2010). Pathology and pathogenesis of ovine pulmonary adenocarcinoma. $J$. Comp. Pathol. 142: 260-283.http://dx.doi.org/10.1016/j.jppa.2009.12.013

Hofacre A and Fan H (2010). Jaagsiekte sheep retrovirus biology and oncogenesis. Viruses 2: 2618-2648. http://dx.doi. org $/ 10.3390 / \mathrm{v} 2122618$

Hopwood P, Wallace WA, Cousens C, Dewar P, et al. (2010). Absence of markers of betaretrovirus infection in human pulmonary adenocarcinoma. Hum. Pathol. 41: 1631-1640. http://dx.doi.org/10.1016/j.humpath.2010.05.013

Johnson ES (1994). Cancer mortality among workers in the meat department of supermarkets. Occup. Environ. Med. 51: 541-547.http://dx.doi.org/10.1136/oem.51.8.541

Johnson ES (2011). Cancer mortality in workers employed in cattle, pigs, and sheep slaughtering and processing plants. Environ. Int. 37: 950-959. http://dx.doi.org/10.1016/j.envint.2011.03.014

Leroux C, Girard N, Cottin V, Greenland T, et al. (2007). Jaagsiekte sheep retrovirus (JSRV): from virus to lung cancer in sheep. Vet. Res. 38: 211-228.http://dx.doi.org/10.1051/vetres:2006060

Lewis FI, Brülisauer F, Cousens C, McKendrick IJ, et al. (2011). Diagnostic accuracy of PCR for Jaagsiekte sheep retrovirus using field data from 125 Scottish sheep flocks. Vet. J. 187: 104-108. http://dx.doi.org/10.1016/j.tvjl.2009.10.024

Linnerth-Petrik NM, Walsh SR, Bogner PN, Morrison C, et al. (2014). Jaagsiekte sheep retrovirus detected in human lung cancer tissue arrays. BMC Res. Notes 7: 160. http://dx.doi.org/10.1186/1756-0500-7-160

Liu SL and Miller AD (2007). Oncogenic transformation by the jaagsiekte sheep retrovirus envelope protein. Oncogene 26: 789-801.http://dx.doi.org/10.1038/sj.onc.1209850

McLean D and Pearce N (2004). Cancer among meat industry workers. Scand. J. Work, Environ. Health 30: 425-437. http://dx.doi.org/10.5271/sjweh.831

Palmarini M and Fan H (2001). Retrovirus-induced ovine pulmonary adenocarcinoma, an animal model for lung cancer. J. Natl. Cancer Inst. 93: 1603-1614. http://dx.doi.org/10.1093/jnci/93.21.1603

Palmarini M and Fan H (2003). Molecular biology of jaagsiekte sheep retrovirus. Curr. Top. Microbiol. Immunol. 257: 81-115. http://dx.doi.org/10.1007/978-3-642-55638-8_4

Palmarini M, Hallwirth C, York D, Murgia D, et al. (2000). Molecular cloning and functional analysis of three type D endogenous retroviruses of sheep reveal a different cell tropism from that of the highly related exogenous jaagsiekte sheep retrovirus. J. Virol. 74: 8065-8076. http://dx.doi.org/10.1128/JVI.74.17.8065-8076.2000

Qi JW, Wu XL, Liu SY and Cao SF (2012). Expression of endogenous beta retroviruses and Hyal-2 mRNA in immune organs of fetuses and lambs. Virol. Sin. 27: 83-92. http://dx.doi.org/10.1007/s12250-012-3222-4

Genetics and Molecular Research 15 (3): gmr.15038521 
Rocca S, Sanna MP, Leoni A, Cossu A, et al. (2008). Presence of Jaagsiekte sheep retrovirus in tissue sections from human bronchioloalveolar carcinoma depends on patients' geographical origin. Hum. Pathol. 39: 303-304. http://dx.doi. org/10.1016/j.humpath.2007.10.008

Salvatori D, González L, Dewar P, Cousens C, et al. (2004). Successful induction of ovine pulmonary adenocarcinoma in lambs of different ages and detection of viraemia during the preclinical period. J. Gen. Virol. 85: 3319-3324. http:// dx.doi.org/10.1099/vir.0.80333-0

Sharp JM and DeMartini JC (2003). Natural history of JSRV in sheep. Curr. Top. Microbiol. Immunol. 275: 55-79. http:// dx.doi.org/10.1007/978-3-642-55638-8_3

Sollner J, Grohmann R, Rapberger R, Perco P, et al. (2008). Analysis and prediction of protective continuous B-cell epitopes on pathogen proteins. Immunome Res. 4: 1. http://dx.doi.org/10.1186/1745-7580-4-1

Spencer TE, Mura M, Gray CA, Griebel PJ, et al. (2003). Receptor usage and fetal expression of ovine endogenous betaretroviruses: implications for coevolution of endogenous and exogenous retroviruses. J. Virol. 77: 749-753. http://dx.doi.org/10.1128/JVI.77.1.749-753.2003

Summers C, Neill W, Dewar P, Gonzalez L, et al. (2002). Systemic immune responses following infection with Jaagsiekte sheep retrovirus and in the terminal stages of ovine pulmonary adenocarcinoma. J. Gen. Virol. 83: 1753-1757. http:// dx.doi.org/10.1099/0022-1317-83-7-1753

Sun S, Schiller JH and Gazdar AF (2007). Lung cancer in never smokers- a different disease. Nat. Rev. Cancer 7: 778-790. http://dx.doi.org/10.1038/nrc2190

Wootton SK, Halbert CL and Miller AD (2005). Sheep retrovirus structural protein induces lung tumours. Nature 434: 904-907.http://dx.doi.org/10.1038/nature03492

Yang J, Yan R, Roy A, Xu D, et al. (2015). The I-TASSER Suite: protein structure and function prediction. Nat. Methods 12: 7-8. http://dx.doi.org/10.1038/nmeth.3213

Yousem SA, Finkelstein SD, Swalsky PA, Bakker A, et al. (2001). Absence of jaagsiekte sheep retrovirus DNA and RNA in bronchioloalveolar and conventional human pulmonary adenocarcinoma by PCR and RT-PCR analysis. Hum. Pathol. 32: 1039-1042.http://dx.doi.org/10.1053/hupa.2001.28249 